\title{
A RATIONAL BILLIARD FLOW IS UNIQUELY ERGODIC IN ALMOST EVERY DIRECTION
}

\author{
BY STEVEN KERCKHOFF, HOWARD MASUR AND JOHN SMILLIE ${ }^{1}$
}

The nature of the long-term behavior of a billiard ball moving on a frictionless table is a question with strong intuitive appeal. A billiard flow is a type of geodesic flow. Geodesic flows, and billiard flows in particular, have provided important examples in the field of dynamical systems.

Let $Q$ be a planar polygon. One can define a geodesic flow $f_{t}$ on the unit tangent bundle $U(Q)$ so that orbits of this flow project to billiard ball paths on $Q$. The polygon $Q$ is said to be rational if all of the angles of $Q$ are rational multiples of $\pi$. When $Q$ is rational the tangent vectors to a given orbit are parallel to a finite set of unit vectors. The orbits with initial direction $\theta$ lie in an invariant surface $M_{\theta} . M_{\theta}$ consists of a finite number of copies of $Q$, one for each potential direction of an orbit with initial direction $\theta$ (cf. [F-K]). The dynamical analysis of $f_{t}$ breaks up into an analysis of the flows $f_{t} \mid M_{\theta}$ as $\theta$ varies.

The results of $[\mathbf{Z}-\mathbf{K}]$ and $[\mathbf{B}-\mathbf{K}-\mathbf{M}]$ show that for a typical direction $\theta$ the flow is minimal, i.e. all orbits are dense. The purpose of this note is to announce the following

THEOREM 1. For almost every $\theta$ the flow $f_{t} \mid M_{\theta}$ is uniquely ergodic.

A flow is ergodic with respect to a probability measure if every invariant set has measure zero or one. A flow is uniquely ergodic if there is precisely one invariant probability measure. A uniquely ergodic flow is ergodic with respect to its unique invariant measure. The surfaces $M_{\theta}$ described above have natural invariant measures coming from Lebesgue measure on $Q$. Theorem 1 implies that the billiard flows are ergodic in almost every direction with respect to the natural invariant measures.

Unique ergodicity can also be described in terms of the distribution of orbits. An orbit is uniformly distributed with respect to a probablity measure $\mu$ if for every open set $V$ with $\mu(\partial V)=0$ the orbit visits $V$ with an asymptotic frequency of $\mu(V)$. A flow is uniquely ergodic if every orbit is uniformly distributed. The natural measures on the sets $M_{\theta}$ project to Lebesgue measure on $Q$. These remarks imply the following

COROLlaRY 1. For almost every $\theta$ the projection to $Q$ of every orbit with initial direction $\theta$ is uniformly distributed in $Q$.

Theorem 1 has consequences for billiard tables which do not have rational angles. The set of all polygons with a given number of sides forms an open

Received by the editors October 31, 1984.

1980 Mathematics Subject Classification. Primary 70D99, 58F11, 30F30.

${ }^{1}$ This work was supported by the National Science Foundation, the Mathematical Sciences Research Institute and the Institute for Advanced Study. 
subset of a finite-dimensional vector space. We would like to thank A. Katok and M. Boshernitzan for pointing out the following corollary to Theorem 1.

COROLlaRY 2. There is a dense $G_{\delta}$ consisting of polygons for which the billiard flow, $f_{t}$, is ergodic.

If $Q$ is a rectangle or, more generally, if reflections through the sides of $Q$ generate a tesselation of the plane then Theorem 1 is a consequence of Weyl's analysis of toral flows, as is pointed out in [F-K].

If the affine group generated by reflections in the sides of $Q$ acts discretely on the plane then Theorem 1 follows from results in $[\mathbf{B}$ and $\mathbf{G}]$.

Theorem 1 follows from a result that we prove about Riemann surfaces and quadratic differentials. A quadratic differential $q$ determines a "real foliation" defined by $\operatorname{Re} q^{1 / 2} d z=0$. This foliation admits a transverse invariant measure. If it admits precisely one such measure we say that it is uniquely ergodic.

THEOREM 2. Given a compact Riemann surface $M$ and a holomorphic quadratic differential $q$ then for almost all $\theta$ the real foliation of $e^{i \theta} q$ is uniquely ergodic.

The unique ergodicity of almost all interval exchanges proved in [ $\mathbf{M}$ and V] follows from Theorem 2. The proof of Theorem 2 uses a result from $[\mathbf{M}]$ relating the unique ergodicity of $q$ to the asymptotic behavior of $q$ under the Teichmüller flow.

\section{REFERENCES}

[B-K-M] C. Boldrighini, M. Keane and F. Marchetti, Billiands in polygons, Ann. Prob. 6 (1978), 532-540.

[B] M. Boshernitzan, A condition for minimal interval exchange maps to be uniquely ergodic, preprint.

[F-K] R. H. Fox and R. B. Kershner, Concerning the transitive properties of geodesics on a rational polyhedrom, Duke Math. J. 2 (1936), 147-150.

[G] E. Gutkin, Billiards on almost integrable polyhedral surfaces, preprint.

[M] H. Masur, Interval exchange tranformations and measured foliations, Ann. of Math (2) 115 (1982), 169-200.

[V] W. A. Veech, Gauss measures for transformations on the space of interval exchange maps, Ann. of Math. (2) 115 (1982), 201-242.

[Z-K] A. N. Zemlyakov and A. B. Katok, Topological transitivity of billiards in polygons, Mat. Zametki 18 (1975), 291-300 (English translation in Mat. Notes 18 (1976), 760-764).

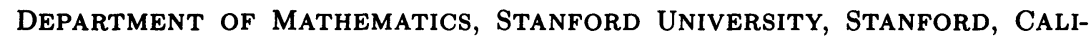
FORNIA 94305

DEPARTMENT OF MATHEMATICS, UNIVERSity OF Illinois at Chicago, ChicAgo, ILlinois 60680

Department of Mathematics, lehman College, the BronX, NeW York 10468

Department of Mathematics, Graduate Center, NeW York, NeW York 10036 